\title{
Análisis de las transformaciones en las coberturas del humedal urbano Tibanica, localidad de Bosa, Bogotá D. C.
}

Coverage Transformations Analysis of Tibanica Urban Wetland, Locality of Bosa, Bogotá D. C.

Análise das transformações nas coberturas do pantanal urbano Tibanica, localidade de Bosa, Bogotá D. C.

Catalina Fuentes Nieto*

Cristian Camilo López Velandia*

Recibido: 3 de junio de 2019

Aprobado: 19 de diciembre de 2019

Doi: https://doi.org/10.12804/revistas.urosario.edu.co/territorios/a.7951

Para citar este artículo:

Fuentes Nieto, C., \& López Velandia, C. C. (2020). Análisis de las transformaciones en las coberturas del humedal urbano Tibanica, localidad de Bosa, Bogotá D. C. Territorios (43), 1-24. https://doi. org/10.12804/revistas.urosario.edu.co/territorios/a.7951

\begin{abstract}
* Docente en la Secretaría de Educación de Bogotá (Colombia). Correo electrónico: cafuentesn@ educacionbogota.edu. co. ORCID: http://orcid. org/0000-0001-90323820

** Coordinador Nacional de Desarrollo Curricular, Fundación Universitaria del Área Andina; docente de cátedra, Maestría en Hidrogeología Ambiental, Universidad Antonio Nariño. Correos electrónicos: clopez163@ areandina.edu.co, crlopez66@uan.edu.co. ORCID: http://orcid. org/0000-0003-30888031
\end{abstract}


Palabras clave

Humedales urbanos; coberturas de la tierra; estadistica multivariante; Bogotá; Tibanica.

Keywords

Urban wetlands; land cover; multivariate statistics; Bogotá; Tibanica.

Palavras-chave

Pantanais urbanos; coberturas da

terra; estatística multivariada; Bogotá; Tibanica.

\section{territarias 43}

\section{RESUMEN}

El presente estudio analizó las trasformaciones en las coberturas de la tierra desarrolladas en el humedal urbano Tibanica, a través de la identificación, clasificación y cuantificación de las coberturas, empleando fotografías aéreas de los años 1993 a 2015. Los resultados indican un predominio de las coberturas asociadas con el accionar humano, tales como zonas verdes urbanas y tejido urbano continuo, y una disminución en aquellas relacionadas con áreas naturales, tales como los cuerpos de agua. Del análisis estadístico, se corroboró la asociación negativa entre el aumento de la población y el área urbanizada, así como la reducción de las coberturas de tipo más natural, lo que evidencia que el incremento en los procesos de urbanización en el área de estudio ha llevado a la reducción de coberturas, tales como lagos, lagunas y ciénagas, pastos limpios, canales y vegetación secundaria.

\section{ABSTRACT}

The present study analyzed the land coverage transformations developed in the Tibanica urban wetland, through the identification, classification and quantification of land coverage using aerial photographs from 1993 to 2015. The results indicate a predominance of the coverages associated with human actions such as urban green areas and continuous urban fabric, and a decrease in those related to natural areas such as water bodies. From statistical analysis, the negative association between the population increase and urbanized area, also the reduction of the more natural type of coverage was corroborated, evidencing that urbanization processes increase in the study area has led the coverage reduction, such as lakes, lagoons and swamps, clean pastures, channels and secondary vegetation.

\section{RESUMO}

O presente estudo, analisou as transformações nas coberturas da terra desenvolvidas no pantanal urbano Tibanica, através da identificação, classificação e quantificação das coberturas empregando fotografias aéreas dos anos 1993 a 2015. Os resultados indicam um predomínio das coberturas associadas com o acionar humano tais como zonas verdes urbanas e tecido urbano contínuo, e uma diminuição em aquelas relacionadas com áreas naturais tais como os corpos de água. Da análise estatística, corroborou-se a associação negativa entre o aumento da população e a área urbanizada, assim como a redução das coberturas de tipo mais natural, o que evidencia que o aumento nos processos de urbanização na área de estudo tem levado à redução de coberturas, tais como lagos, laguna e pântanos, pastagens limpas, canais e vegetação secundária. 


\section{Introducción}

Los cambios a escala mundial generados sobre las coberturas de la tierra y extensión de los humedales han sido de grandes magnitudes, estimándose que la pérdida para el siglo XX a nivel global fue entre un $64 \%$ y un $71 \%$, mientras que en algunas regiones costeras y de Asia se presentaron pérdidas de mayor magnitud (Davidson, 2014). Los principales factores asociados a esta problemática guardan relación directa con el desarrollo de infraestructuras (represas, diques y canales), conversión de tierras, extracción de agua, introducción de especies invasoras y la contaminación; adicionalmente, el crecimiento poblacional y el incremento del desarrollo económico son a su vez generadores indirectos de impacto sobre estos ecosistemas (Millenium Ecosystem Assessment, 2005; Olsen, 2000). Estos impulsores de cambio en sus diferentes escalas espaciales y temporales llevan a la disminución de los servicios prestados por los humedales, afectando directamente el bienestar de las personas que habitan estos territorios (Mosquera et al., 2015).

En un contexto urbano, estos ecosistemas han quedado insertos en las ciudades debido a su expansión física y al aumento en la demanda de tierras, además de ser afectados por rellenos, desecamiento, cambios en su vegetación y contaminación de sus aguas (Ramsar, 2018b; Rojas et al., 2013), así como ser considerados como un obstáculo para el desarrollo de la infraestructura urbanística; sin embargo, esta tendencia se ha modificado progresivamente, considerándose a los humedales urbanos como nuevos paradigmas en el contexto de la ecología y la conservación (Van der Hammen et al., 2008), al ser apreciados por la generación de numerosos servicios ecosistémicos, entre los que se encuentran la reducción de ruido, la oferta de espacios para la recreación y cultura, la mejora de la calidad de aire en las ciudades y promover el bienestar humano (Bolund \& Hunhammar, 1999; Pedersen et al., 2019; Ramsar, 2018a). De igual manera, servir de amortiguadores climáticos (Zhang et al., 2015), incluso han sido entendidos como los ecosistemas de mayor valor por hectárea (Costanza et al., 1997).

Para determinar las alteraciones que estos humedales urbanos experimentan, el monitoreo a través del uso de sensores remotos y la comparación entre las imágenes generadas, llevando a cabo una previa identificación y clasificación de coberturas, permite detectar las causas particulares de estos sucesos y a su vez analizar los efectos que se hayan producido a lo largo del tiempo (Camilleri et al., 2017; Chuvieco, 1995, 2016; Hoyos et al., 2018). Particularmente, las fotografías aéreas pueden ser empleadas para identificar, delinear y medir de forma acertada la extensión de los humedales urbanos (Jiang et al., 2012), cuya información se constituye como un 
insumo para apoyar la toma de decisiones en los procesos de planificación y manejo de estos ecosistemas (Rebelo et al., 2009).

En Colombia, los humedales urbanos presentan procesos significativos de cambio causados directa o indirectamente por los patrones de distribución de los asentamientos humanos en el país (Ministerio del Medio Ambiente, 2002), los cuales se han localizado principalmente en las regiones Andina, Caribe y al suroccidente del país, mediante una progresiva conurbación y metropolización alrededor de las grandes ciudades (Ministerio de Ambiente, Vivienda y Desarrollo Territorial, 2008). Sumado a lo anterior, se encuentra que la falta de conocimiento sobre la función, composición y valor de los humedales, la concepción negativa de espacios insalubres, peligrosos y opuestos al desarrollo (común entre las culturas de montaña), representan condiciones que afectan las conservación de estos ecosistemas (Van der Hammen et al., 2008).

Particularmente en la capital, Bogotá D. C., se presenta una alteración en la estructura y función de todos los humedales presentes en el territorio, debido a cambios en la cobertura y uso de la tierra, como consecuencia de su progresiva incorporación al desarrollo urbano e industrial (Van der Hammen et al., 2008). Un ejemplo de esta situación se evidencia en el humedal Tibanica, ubicado en la localidad de Bosa, el cual ha presentado una reducción significativa en la cantidad y calidad del agua, debido a su nula movilidad y a la acumulación de residuos provenientes de las actividades humanas que lo rodean. Adicionalmente, se presenta una disminución en la diversidad de coberturas naturales, causada principalmente por la presión de la expansión urbana, generando la transformación casi total del paisaje (Secretaría Distrital de Ambiente \& Instituto de Estudios Ambientales, 2006). A pesar de la aplicación de múltiples estrategias de gestión para mejorar las condiciones del humedal, no se ha logrado un retroceso en su proceso de deterioro (Contraloría de Bogotá, 2010).

Los humedales de Bogotá han sido objeto de varios estudios que han contemplado el análisis multitemporal de imágenes satelitales y fotografías aéreas, como los realizados por Cardona y Pinilla (2015) y Ruiz (2015), quienes identificaron los cambios en las coberturas en cuanto a su área. Ordóñez, González y Cardona (2016) emplearon esta metodología para la generación de estrategias que permitan la conectividad entre tres humedales del Distrito Capital, y Mayorga (2016) relacionó la pérdida de área en los humedales de la localidad de Kennedy con los procesos de urbanización, desde un enfoque normativo.

De acuerdo con lo anterior, se planteó este estudio con el objetivo de: 1) determinar los procesos de transformación de las coberturas presentes en el humedal urbano Tibanica en el período 1993-2015;

\section{territorias 43}


y 2) identificar la relación de la actividad antrópica asociada al desarrollo urbano y el crecimiento poblacional sobre las dinámicas de las coberturas en el área de estudio.

\section{Área de estudio}

El humedal de Tibanica se encuentra ubicado en la localidad séptima de Bosa, en la UPZ 85, Bosa Central, en el Distrito Capital, al occidente de la Autopista Sur y al suroccidente de Bogotá, en límite con el municipio de Soacha, entre las coordenadas extremas N4 $36^{\circ} 22,4028^{\prime \prime}$ - N4³5'59,1864" y W74¹2'33,5232” W74 12 '4,3272"; tiene una cobertura de 28,8 ha, según la Resolución 194 de 1995 de la EAAB (Alcaldía Mayor de Bogotá \& Departamento Técnico Administrativo del Medio Ambiente, 2006). Limita al norte con los barrios Manzanares y La Esperanza de Tibanica, al oriente con la urbanización Alameda del Parque, al occidente con el canal Tibanica, que define el límite del Distrito, y al sur con el municipio de Soacha (figura 1). Este humedal se constituye como el mayor referente de espacio público verde para la localidad de Bosa, la cual se ubica por debajo del promedio de Bogotá y además se encuentra en una de las zonas más secas de la capital del país (Departamento Administrativo de la Defensoría del Espacio Público, 2017; SDA \& IDEA, 2006).
El área de estudio suprayace las formaciones del Cuaternario entre las que resaltan la Formación Sabana (Qsa) y los depósitos aluviales y coluviones, compuestos por arenas, gravas y arcillas (Lobo Guerrero, 1992; Montoya \& Reyes, 2005). En un contexto geomorfológico, el humedal se encuentra a lo largo de los meandros formados en la planicie aluvial del río Tunjuelito generados por la erosión lateral de la corriente, siendo algunas de estas áreas utilizadas por cultivos, rellenos y actividades urbanísticas. Otra geoforma importante corresponde a bajos o depresiones inundables (humedales), la cual fue transformada por procesos de relleno para la construcción de urbanizaciones (barrios Los Olivos y Manzanares) (Moreno, 1995). El régimen de precipitación en el humedal Tibanica presenta una distribución de carácter bimodal, caracterizada por dos períodos de bajas precipitaciones entre los meses de diciembre a febrero $(35,2 \pm$ $31,9 \mathrm{~mm}$; estación INEM Kennedy) y junio a septiembre $(44,3 \pm 23,4 \mathrm{~mm}$; estación INEM Kennedy), y dos períodos de altas precipitaciones entre los meses de marzo a mayo $(81,0 \pm 44,7 \mathrm{~mm}$; estación INEM Kennedy) y octubre a noviembre $(82,4 \pm$ 39,7 mm; estación INEM Kennedy). La temperatura muestra un comportamiento estable con una media de $12,0 \pm 0,1^{\circ} \mathrm{C}$ (estación San Jorge GJA).

Conforme con el estudio realizado por Moreno (1995), en el cual se analizaron las actividades antrópicas y su efecto en 


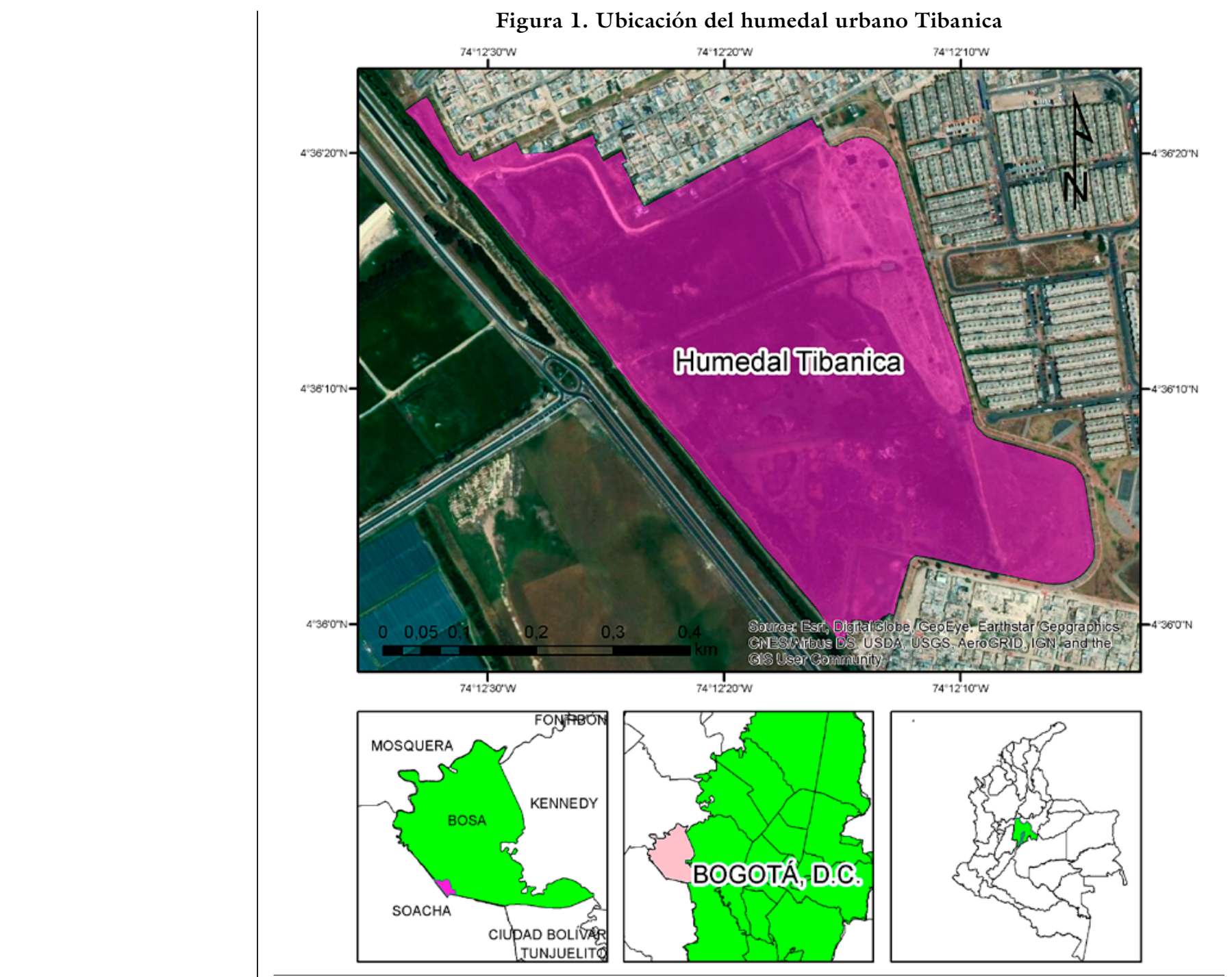

Fuente: elaboración de los autores.

la tranformación de las coberturas en el $\mid$ de diques artificiales en su interior, así humedal Tibanica para el período de 1940 hasta 1995 , se destaca la construcción como la disminución progresiva de su área gracias a los procesos de relleno para la 
edificación de barrios en estratos socioeconómicos 1 y 2 , que en su mayoría tienen un origen ilegal, y que posteriormente fueron legalizados por parte de la comunidad.

\section{Metodología}

Se llevó a cabo un análisis multitemporal para determinar los cambios en las coberturas de la tierra del humedal Tibanica entre los años 1993, 1999, 2004, 2009 y 2015, empleando ortofotomosaicos de la ciudad de Bogotá y fotografías aéreas (tabla 1). Las aerofotografías fueron georreferenciadas empleando el método de modelo polinomial de primer orden y proyectadas al sistema de proyección Magna-Sirgas-Colombia-Bogotá.

Posteriormente, se llevó a cabo la creación de la leyenda, definiendo las clases de cobertura de acuerdo con la metodología Corine Land Cover adaptada para Colombia (Ideam, 2010), teniendo en cuenta las unidades de clasificación en los niveles 1, 2 y 3 para el humedal Tibanica (tabla 2). Esta metodología tuvo como propósito la realización del inventario homogéneo de la cobertura de la superficie de la tierra a partir de la interpretación visual de imágenes de satélite asistida por computador y la generación de una base de datos geográfica.

Se efectuó la comparación de imágenes previamente clasificadas de manera independiente, a través de la digitalización en forma vectorial de cada una de las coberturas presentes en el humedal Tibanica, permitiendo calcular sus diferentes áreas, siguiendo la metodología propuesta por Franco (2016). Se llevó a cabo el geoprocesamiento por medio de la intersección de capas de información de los distintos períodos, el cálculo de áreas, la concatenación de campos en tablas y el consolidado de tablas o summarize empleando el software ArcGis 10.5. A

Tabla 1. Material cartográfico empleado para el análisis multitemporal del humedal Tibanica

\begin{tabular}{|l|l|l|}
\hline Años & \multicolumn{1}{|c|}{ Tipo de material } & \multicolumn{1}{|c|}{ Entidad de origen } \\
\hline 1993 & Aerofotografía pancromática. Vuelo C-2520-113 & \multirow{2}{*}{$\begin{array}{c}\text { Instituto Geográfico Agustín Codazzi } \\
\text { (IGAC). Licencia de uso 4641-2017 }\end{array}$} \\
\hline 1999 & Aerofotografía pancromática. Vuelo C-2654-167 & \\
\hline 2004 & Aerofotografía pancromática. Vuelo C-2717-185 & $\begin{array}{l}\text { Portal de infraestructura de datos espa- } \\
\text { ciales para el Distrito Capital (IDECA) }\end{array}$ \\
\hline $\begin{array}{l}2011 \\
2004\end{array}$ & Ortofotografías de la ciudad de Bogotá & \\
\hline
\end{tabular}

Fuente: elaboración de los autores. 
Tabla 2. Unidades empleadas para la clasificación de coberturas para el humedal Tibanica

\begin{tabular}{|l|l|l|}
\hline \multicolumn{1}{|c|}{ Nivel 1 } & \multicolumn{1}{|c|}{ Nivel 2 } & \multicolumn{1}{c|}{ Nivel 3 } \\
\hline \multirow{2}{*}{ 1. Territorios artificializados } & 1.1. Zonas urbanizadas & $\begin{array}{l}\text { 1.1.1. Tejido urbano continuo } \\
\text { 1.1.2. Tejido urbano discontinuo }\end{array}$ \\
\cline { 2 - 3 } & $\begin{array}{l}\text { 1.4. Zonas verdes artificializadas, } \\
\text { no agrícolas }\end{array}$ & 1.4.1. Zonas verdes urbanas \\
\hline 2. Territorios agrícolas & 2.3. Pastos & 2.3.1. Pastos limpios \\
\hline 3. Bosques y áreas seminaturales & $\begin{array}{l}\text { 3.2. Áreas con vegetación } \\
\text { herbácea y/o arbustiva }\end{array}$ & $\begin{array}{l}\text { 3.2.3. Vegetación secundaria o en } \\
\text { transición }\end{array}$ \\
\hline 4. Áreas húmedas & 4.1. Áreas húmedas continentales & $\begin{array}{l}\text { 4.1.4. Vegetación acuática sobre } \\
\text { cuerpos de agua }\end{array}$ \\
\hline 5. Superficies de agua & 5.1. Aguas continentales & $\begin{array}{l}5.1 .2 . \text { Lagunas, lagos y ciénagas } \\
\text { naturales } \\
5.1 .3 . \text { Canales }\end{array}$ \\
\hline
\end{tabular}

Fuente: Ideam (2010), adaptado por los autores.

partir de estos consolidados de tablas, se construyeron las matrices de transformación en porcentaje de área relativa para los períodos estudio, con el fin de identificar la proporción de conversión entre coberturas.

Se identificaron las relaciones de interdependencia entre las variables, las cuales corresponden a las áreas de cada una de las coberturas interpretadas en el humedal Tibanica y la población de la localidad de Bosa para los años 1993, 1999, 2004, 2009 y 2015, obtenida a partir de los datos provenientes del DANE y la SDP, para lo cual se usó el test de esfericidad de Bartlett a fin de determinar si la matriz de datos es significativamente diferente de una matriz de identidad, es decir, si las variables no se relacionan $\mathrm{y}$, por lo tanto, son inadecuadas para la detección de estructuras (Bartlett, 1937). El análisis de componentes principales (ACP) fue desarrollado usando el software $\mathrm{R}$ (R Core Team, 2017) y la librería FactoClass (Pardo \& Del Campo, 2007).

\section{Resultados}

La tabla 3 expone los estadísiticos sumarios de las áreas de cada una de las coberturas identificadas en el humedal Tibanica, junto con la población de la localidad de Bosa.

En general, se determina que las variables TUC $(32,90 \pm 9,91$ ha) y ZVU $(20,65 \pm$ 4,99 ha) son las coberturas de la tierra más dominantes en el área de estudio. Adicionalmente, la variabilidad en la desviación 
Tabla 3. Datos de áreas (ha) y población total de la localidad de Bosa

\begin{tabular}{|c|c|c|c|c|c|}
\hline Variable & $\bar{X}$ & $\sigma$ & mín. & máx. & $\gamma_{1}$ \\
\hline LL & 1,52 & 1,54 & 0,06 & 3,64 & 0,26 \\
\hline VA & 18,87 & 3,25 & 15,84 & 24,34 & 0,76 \\
\hline TUC & 32,90 & 9,91 & 23,46 & 46,12 & 0,29 \\
\hline ZVU & 20,65 & 4,99 & 15,66 & 27,32 & 0,17 \\
\hline TUD & 2,00 & 4,48 & 0,00 & 10,01 & 1,07 \\
\hline C & 1,24 & 0,23 & 0,88 & 1,52 & $-0,39$ \\
\hline PL & 7,88 & 2,75 & 3,32 & 10,58 & $-0,69$ \\
\hline VS & 9,70 & 0,71 & 8,53 & 10,39 & $-0,67$ \\
\hline POB & 465746 & 170973 & 215816 & 646833 & $-0,38$ \\
\hline
\end{tabular}

Nota: X: media; $\sigma$ : desviación estándar; mín.: valor mínimo; máx.: valor máximo; $\gamma_{1}$ : coeficiente de asimetría de Fisher; LL: lagunas, lagos y ciénagas naturales; VA: vegetación acuática; TUC: tejido urbano continuo; ZVU: zonas verdes urbanas; TUD: tejido urbano discontinuo; C: canales; PL: pastos limpios; vs: vegetación secundaria; POB: población localidad.

Fuente: elaboración de los autores.

estándar de algunos datos evidencia que las áreas de estas no presentan un comportamiento homogéneo a nivel temporal.

\section{Procesos de transformación de las coberturas}

Según el análisis desarrollado en el humedal Tibanica y su área de influencia, se evidencia que en el año 1993 la cobertura de mayor área $(26,23 \%$ de la extensión total) corresponde a vegetación acuática sobre cuerpos de agua con 24,34 ha, seguida por zonas verdes urbanas con 20,41 ha, que representa el $22 \%$ del área total (figura 2). Para el año 1999, la vegetación acuática sobre cuerpos de agua disminuye a un $20,46 \%$ (18,98 ha) y se generó una conversión de toda la cobertura tejido urbano discontinuo a tejido urbano continuo (proceso de urbanización), llevando a esta última a ser la de mayor área con 25,37 ha (tabla 4). A partir de este año, el tejido urbano continuo se constituye como la cobertura dominante en la zona de estudio. El área cubierta por agua, representada por la cobertura lagos, lagunas y ciénagas naturales, aumentó de 2,49 ha en 1993 a 3,64 ha en 1999, sin embargo, a partir de este año se presenta una disminución progresiva hasta llegar a menos de una hectárea $(0,15$ ha) en el año 2015.

Entre los años 2004 y 2009 se da el mayor aumento de la cobertura tejido urbano continuo, la cual pasó de 
Figura 2. Coberturas humedal Tibanica año 1993

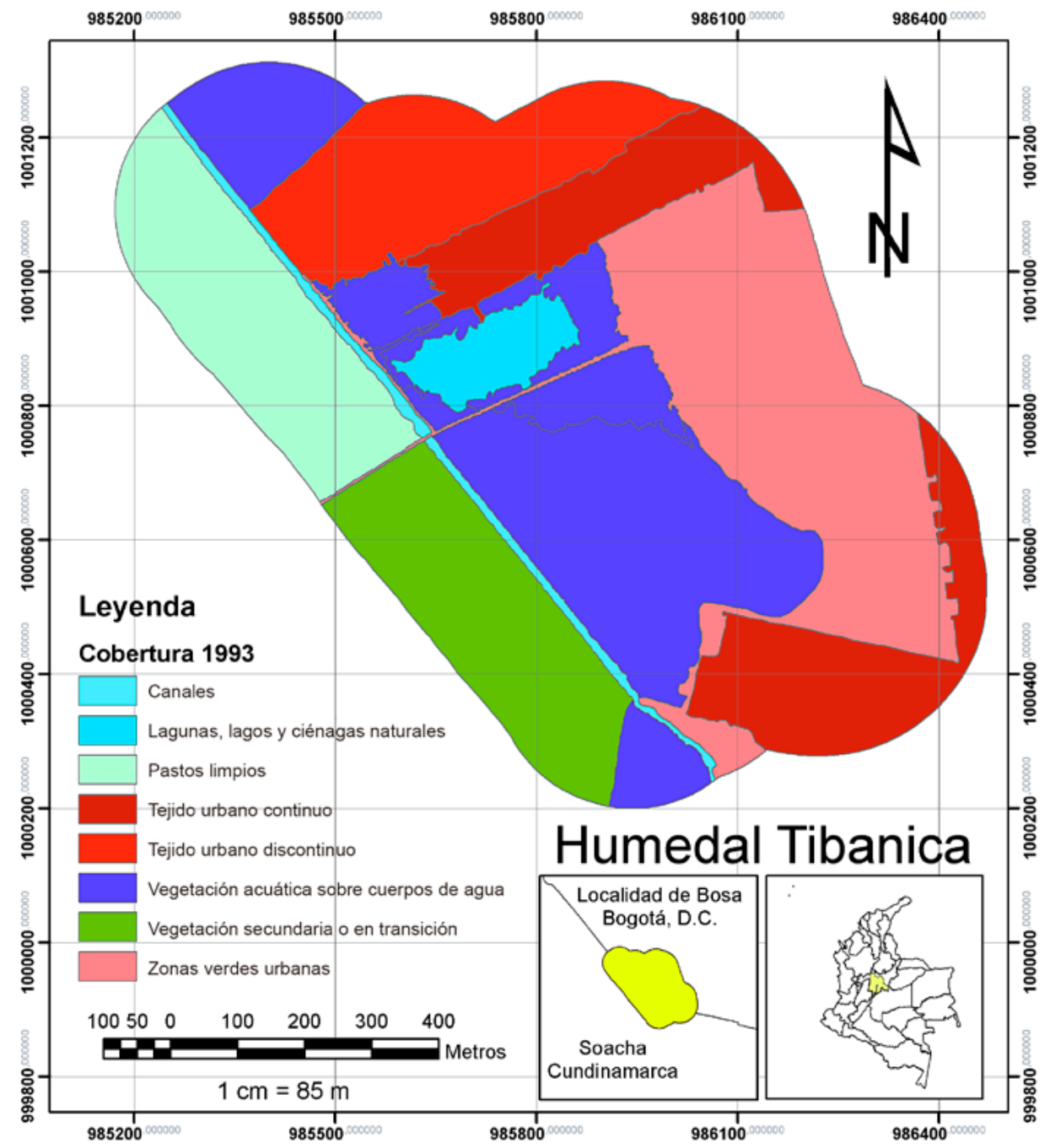

Fuente: elaboración de los autores.

29,06 ha, que equivalen al $31 \%$, a ocupar 40,50 ha, que corresponden al $44 \%$ del área total, debido a la construcción de los conjuntos residenciales Alamedas del
Parque, ubicados en el costado oriental del humedal, que pertenece a la localidad de Bosa (figura 3). 
Tabla 4. Área (ha) y porcentaje (\%) relativo de los tipos de cobertura presentes en el humedal Tibanica para los años 1993, 1999, 2004, 2009 y 2015

\begin{tabular}{|l|c|c|c|c|c|c|c|c|c|c|}
\hline \multicolumn{1}{|c|}{ Cobertura } & $\mathbf{1 9 9 3}$ & $\mathbf{6}$ & $\mathbf{1 9 9 9}$ & $\mathbf{\%}$ & $\mathbf{2 0 0 4}$ & $\mathbf{\%}$ & $\mathbf{2 0 0 9}$ & $\mathbf{\%}$ & $\mathbf{2 0 1 5}$ & $\mathbf{\%}$ \\
\hline $\begin{array}{l}\text { Lagunas lagos y ciénagas } \\
\text { naturales }\end{array}$ & 2,49 & 2,69 & 3,64 & 3,93 & 1,25 & 1,35 & 0,06 & 0,06 & 0,17 & 0,18 \\
\hline $\begin{array}{l}\text { Vegetación acuática sobre } \\
\text { cuerpos de agua }\end{array}$ & 24,34 & 26,23 & 18,98 & 20,46 & 15,84 & 17,08 & 17,58 & 18,95 & 17,63 & 19,01 \\
\hline Tejido urbano continuo & 13,46 & 14,51 & 25,37 & 27,35 & 29,06 & 31,32 & 40,50 & 43,65 & 46,12 & 49,72 \\
\hline Zonas verdes urbanas & 20,41 & 22,00 & 23,76 & 25,61 & 27,32 & 29,44 & 15,66 & 16,89 & 16,12 & 17,37 \\
\hline Tejido urbano discontinuo & 10,01 & 10,79 & 0,00 & 0,00 & 0,00 & 0,00 & 0,00 & 0,00 & 0,00 & 0,00 \\
\hline Canales & 1,52 & 1,64 & 1,29 & 1,39 & 1,21 & 1,30 & 1,30 & 1,40 & 0,88 & 0,94 \\
\hline Pastos limpios & 10,58 & 11,40 & 9,33 & 10,06 & 8,09 & 8,72 & 8,09 & 8,72 & 3,32 & 3,58 \\
\hline $\begin{array}{l}\text { Vegetación secundaria o en } \\
\text { transición }\end{array}$ & 9,96 & 10,73 & 10,39 & 11,20 & 10,01 & 10,79 & 9,59 & 10,33 & 8,53 & 9,19 \\
\hline Total & 92,77 & 100 & 92,77 & 100 & 92,77 & 100 & 92,77 & 100 & 92,77 & 100 \\
\hline
\end{tabular}

Fuente: elaboración de los autores.

Figura 3. Conjuntos Alameda del Parque, construidos al costado oriental del humedal Tibanica

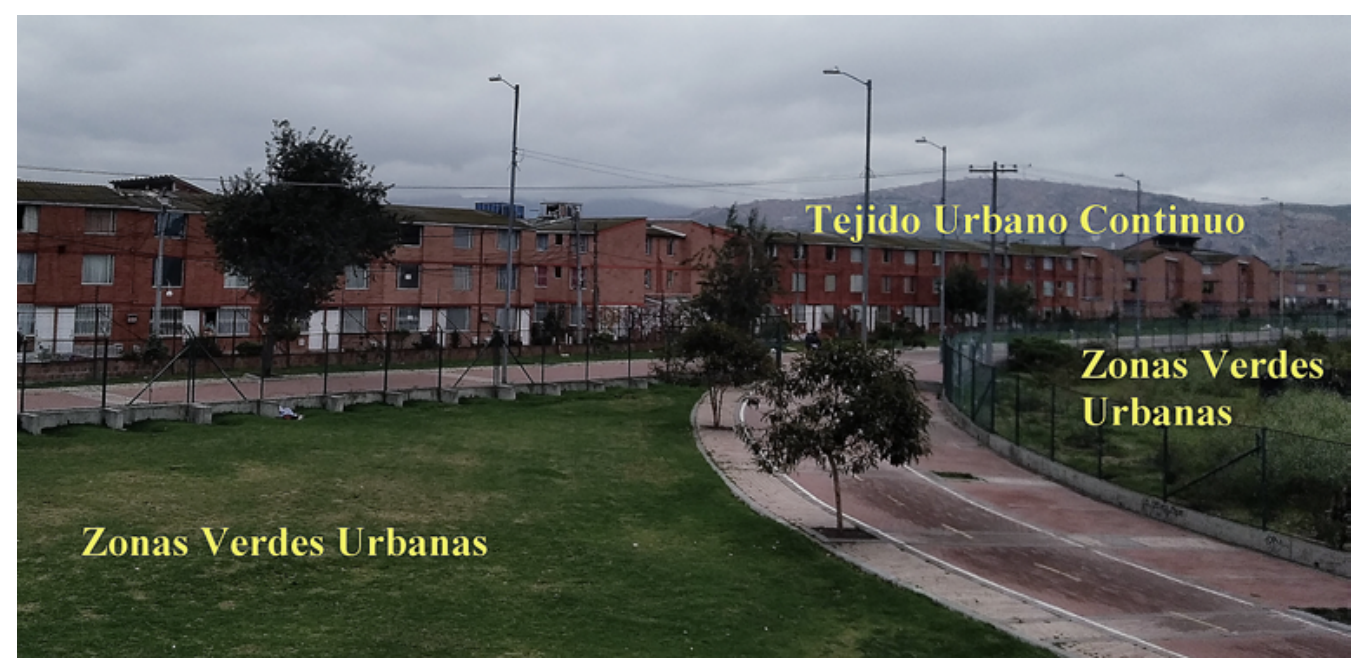

Fuente: elaboración de los autores. 
Las zonas verdes urbanas son áreas cubiertas por vegetación ubicadas dentro o al lado del tejido urbano. Durante el período comprendido entre los años 1993 a 2004, esta cobertura aumentó su área de 20,41 ha $(22 \%)$ a 27,32 ha $(29 \%)$, sin embargo, a partir de 2004 se evidencia una disminución en su área, ocupando 15,66 ha (17\%) en el año 2009 y 16,12 ha en 2015 (17\%). Esta cobertura se encuentra ubicada entre el tejido urbano continuo y el borde de vegetación flotante del humedal Tibani$\mathrm{ca}$, conformando un anillo alrededor de este, el cual presenta como vegetación dominante el pasto kikuyo (Pennisetum clandestinum). En el año 2015, se observa una disminución en las coberturas canales (la cual corresponde al canal Tibanica, que se encuentra paralelo al humedal) de 1,52 ha en 1993 a 0,88 ha en 2014 , pastos limpios (de 10,58 a 3,32 ha) y vegetación secundaria o en transición (de 9,96 a 8,53 ha), que representan en total un $13,7 \%$ del área de estudio. Estas coberturas están situadas en el municipio de Soacha y, para el caso de pastos limpios, estos son empleados para mantenimiento de ganado vacuno (figura 4 ).

\section{Análisis de la dinámica temporal de las coberturas}

Durante el período 1993-2015, se evidencia una transformación del 53,36\% en las coberturas del humedal Tibanica, lo que equivale a 49,50 ha del total de área estudiada. La mayor conversión corresponde a zonas verdes urbanas en tejido urbano continuo, tejido urbano discontinuo en tejido urbano continuo y vegetación acuática en zonas verdes urbanas, que entre las tres suman 30,56 ha $(33 \%)$. Otras conversiones observadas son vegetación acuática por tejido urbano continuo en un $8,49 \%$, pastos limpios a zonas verdes urbanas en un 7,58\% y lagos, lagunas y ciénagas a vegetación acuática en el 5,04\% (tabla 5 y figura 5 ).

En la matriz de transformación (tabla 6) resalta que la cobertura tejido urbano continuo mantiene el $14,45 \%$ de área y se le adiciona un $35,26 \%$ de las coberturas vegetación acuática, zonas verdes urbanas, canales, pastos limpios, vegetación secundaria y tejido urbano discontinuo. La vegetación acuática conserva un $15,78 \%$ de su área original, mientras que lagos, lagunas y ciénagas naturales perdió completamente su área original. Las zonas verdes urbanas mantienen el 4,66\% del área y ha sufrido procesos de transformación en un 12,71\% hacia vegetación acuática, canales, pastos limpios, tejido urbano continuo, vegetación secundaria y tejido urbano discontinuo. Los pastos limpios y la vegetación secundarias son las que menos han recibido aportes de otras coberturas, generalmente ellas se convierten en zonas verdes urbanas y tejido urbano continuo. 
Figura 4. Coberturas del humedal Tibanica para el año 2015

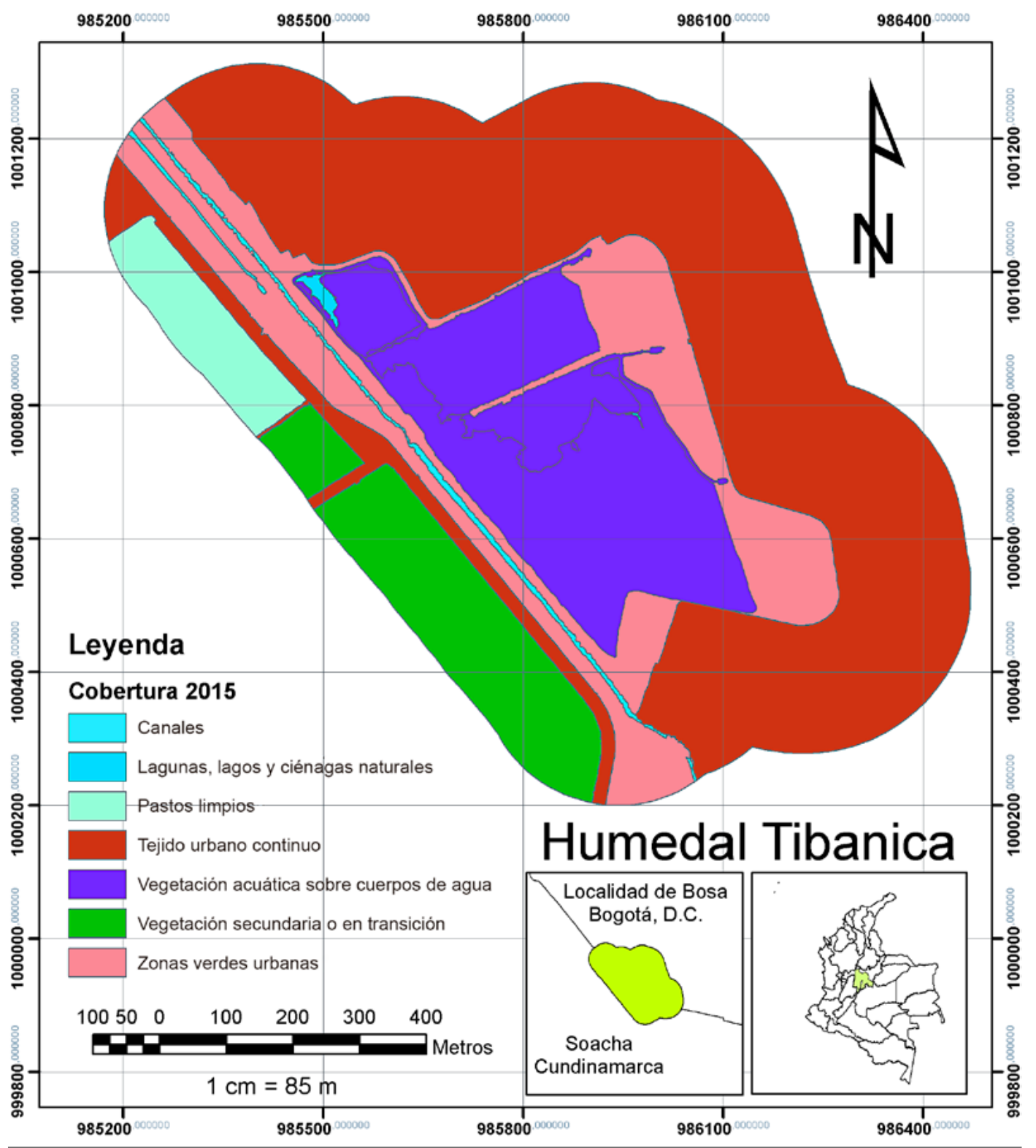

Fuente: elaboración de los autores. 
Tabla 5. Área en hectáreas y porcentaje de las conversiones entre coberturas años 1993-2015

\begin{tabular}{|c|c|c|}
\hline Coberturas & Área 1993-2015 & $\%$ del área \\
\hline Canales - lagunas lagos y ciénagas naturales & 0,0002 & 0,0003 \\
\hline Canales - tejido urbano continuo & 0,09 & 0,19 \\
\hline Canales - vegetación acuática sobre cuerpos de agua & 0,02 & 0,04 \\
\hline Canales - zonas verdes urbanas & 1,22 & 2,46 \\
\hline Lagunas, lagos y ciénagas naturales - vegetación acuática sobre cuerpos de agua & 2,49 & 5,04 \\
\hline Pastos limpios - canales & 0,45 & 0,92 \\
\hline Pastos limpios - tejido urbano continuo & 1,91 & 3,87 \\
\hline Pastos limpios - vegetación secundaria o en transición & 1,14 & 2,30 \\
\hline Pastos limpios - zonas verdes urbanas & 3,75 & 7,58 \\
\hline Tejido urbano continuo - vegetación acuática sobre cuerpos de agua & 0,03 & 0,05 \\
\hline Tejido urbano continuo - zonas verdes urbanas & 0,03 & 0,07 \\
\hline Tejido urbano discontinuo - lagunas, lagos y ciénagas naturales & 0,05 & 0,10 \\
\hline Tejido urbano discontinuo - tejido urbano continuo & 9,45 & 19,08 \\
\hline Tejido urbano discontinuo - vegetación acuática sobre cuerpos de agua & 0,15 & 0,31 \\
\hline Tejido urbano discontinuo - zonas Verdes urbanas & 0,36 & 0,73 \\
\hline Vegetación acuática sobre cuerpos de agua - canales & 0,05 & 0,09 \\
\hline Vegetación acuática sobre cuerpos de agua - lagunas, lagos y ciénagas naturales & 0,12 & 0,23 \\
\hline Vegetación acuática sobre cuerpos de agua - tejido urbano continuo & 4,20 & 8,49 \\
\hline Vegetación acuática sobre cuerpos de agua - zonas verdes urbanas & 5,33 & 10,76 \\
\hline Vegetación secundaria o en transición - canales & 0,19 & 0,38 \\
\hline Vegetación secundaria o en transición - tejido urbano continuo & 1,28 & 2,59 \\
\hline Vegetación secundaria o en transición - zonas verdes urbanas & 1,10 & 2,22 \\
\hline Zonas verdes urbanas - canales & 0,002 & 0,003 \\
\hline Zonas verdes urbanas - lagunas, lagos y ciénagas naturales & 0,01 & 0,01 \\
\hline Zonas verdes urbanas - tejido urbano continuo & 15,78 & 31,88 \\
\hline Zonas verdes urbanas - vegetación acuática sobre cuerpos de agua & 0,30 & 0,60 \\
\hline Total & 49,50 & 53,36 \\
\hline
\end{tabular}

\section{territarios 43}

Fuente: elaboración de los autores. 
Figura 5. Intersección de coberturas del humedal Tibanica años 1993-2015

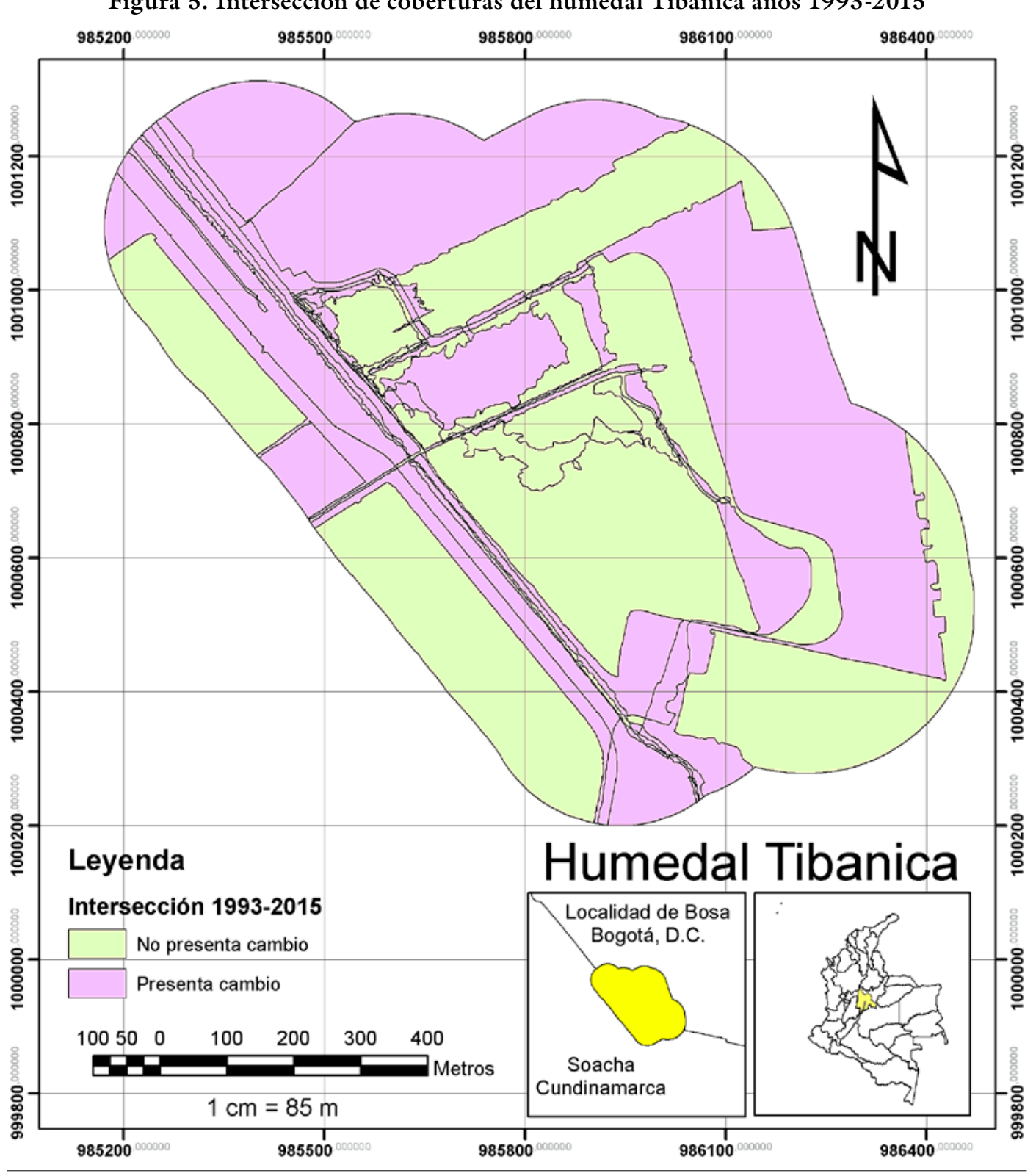

Fuente: elaboración de los autores.

territarias 43

15 
Tabla 6. Matriz de transformación en porcentaje de área relativa período 1993-2015

\begin{tabular}{|c|c|c|c|c|c|c|c|c|c|}
\hline & \multicolumn{9}{|c|}{2015} \\
\hline \multirow{9}{*}{1993} & 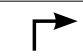 & vA & $\mathrm{ZVU}$ & C & LL & PL & TUC & vs & TUD \\
\hline & VA & 15,78 & 5,74 & 0,05 & 0,12 & & 4,53 & & \\
\hline & ZVU & 0,32 & 4,66 & 0,002 & 0,01 & & 17,01 & & \\
\hline & C & 0,02 & 1,32 & 0,20 & & & 0,10 & & \\
\hline & LL & 2,69 & & & 0 & & & & \\
\hline & PL & & 4,04 & 0,49 & & 3,58 & 2,06 & 1,23 & \\
\hline & TUC & 0,03 & 0,04 & & & & 14,45 & & \\
\hline & vs & & 1,18 & 0,20 & & & 1,38 & 7,96 & \\
\hline & TUD & 0,17 & 0,39 & & & & 10,18 & & 0 \\
\hline
\end{tabular}

Nota: VA: vegetación acuática sobre cuerpos de agua; ZVU: zonas verdes urbanas; C: canales;

LL: lagunas, lagos y ciénagas naturales; PL: pastos limpios; TUC: tejido urbano continuo;

Vs: vegetación secundaria o en transición; TUD: tejido urbano discontinuo.

Fuente: elaboración de los autores.

\section{Análisis de componentes principales}

Previo al análisis, la prueba de esfericidad de Bartlett fue calculada con las áreas de cada una de las coberturas identificadas en el humedal Tibanica, junto con la población de la localidad de Bosa, obteniendo que los datos presentan una diferencia estadísticamente significativa (Bartlett's K-squared $=587,87, \mathrm{df}=8, \mathrm{p}$-value $<2,2$ $\mathrm{x} 10^{-16}$ ), por lo cual la aplicación de un ACP genera una reducción significativa de la dimensionalidad de los datos originales. El ACP fue llevado a cabo por la diagonalización de la matriz de correlación; las variables fueron autoescaladas a media

\section{territarias 43} cero y unidad de varianza, evitando los problemas debido a las diferentes escalas de medición y rangos numéricos variables que presentan los parámetros analizados.

Se encuentra que las dos primeras componentes responden por el $89,79 \%$ de la varianza total (tabla 7 y figura 6), por lo tanto, estas dos componentes son seleccionadas al representar más del $80 \%$ de la variación y sus valores propios (eigenvalues) son mayores a 0,7 .

El primer factor ( $\mathrm{PCl}$ ) explica el 68,4\% del total de la varianza, presentando una fuerte asociación positiva $(>0,95)$ con las variables tejido urbano continuo (TUC) y población ( $\mathrm{POB})$, representando una relación directa entre estas variables. Así mismo, una fuerte asociación negativa $(<-$ $0,85)$ con las variables pastos limpios (PL), 
Figura 6. Varianza acumulada para cada factor

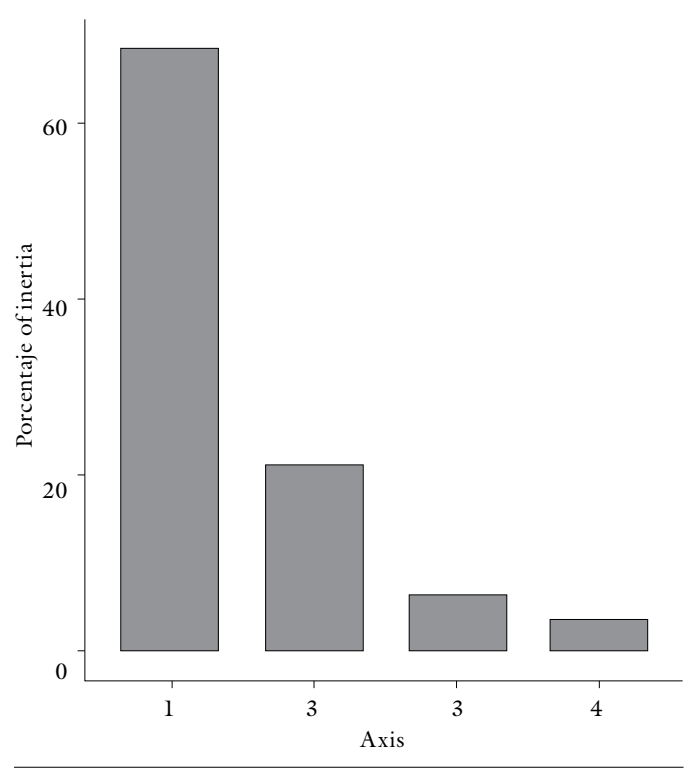

Fuente: elaboración de los autores.

Tabla 7. Valores propios y varianza acumulada para cada factor

\begin{tabular}{|c|c|c|c|}
\hline \multirow{2}{*}{ Eje } & \multirow{2}{*}{$\begin{array}{l}\text { Valores } \\
\text { propios }\end{array}$} & \multicolumn{2}{|c|}{ Inercia proyectada } \\
\cline { 3 - 4 } & $\%$ & Acumulada (\%) \\
\hline 1 & 61,571 & 68,412 & 68,41 \\
\hline 2 & 19,238 & 21,375 & 89,79 \\
\hline 3 & 0,5851 & 6,501 & 96,29 \\
\hline 4 & 0,3341 & 3,712 & 100 \\
\hline
\end{tabular}

Fuente: elaboración de los autores.

canales (C), vegetación secundaria (vs) y lagos y lagunas (LL) (figura 7 y tabla 8), lo que indica una relación inversa entre este grupo de variables; de esta manera,
Figura 7. Diagrama de dispersión de la comparación de los valores para Compl y Comp2

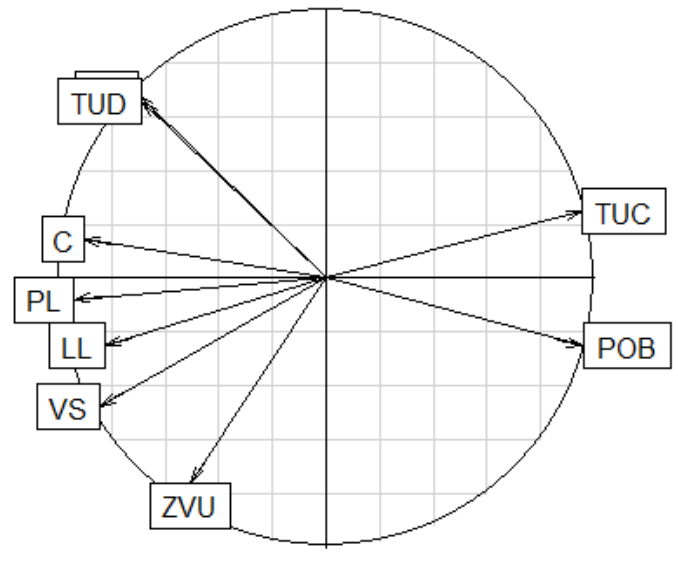

Fuente: elaboración de los autores

Tabla 8. Matriz de coordenadas para las variables asociadas a las componentes principales PC1 y PC2

\begin{tabular}{|c|c|c|}
\hline & PC1 & PC2 \\
\hline LL & $-0,8267063$ & $-0,25066155$ \\
\hline VA & $-0,7034146$ & 0,68538238 \\
\hline TUC & 0,9570551 & 0,24885968 \\
\hline ZVU & $-0,5056309$ & $-0,75998932$ \\
\hline TUD & $-0,6851783$ & 0,65710165 \\
\hline C & $-0,9043388$ & 0,14577969 \\
\hline PL & $-0,9409426$ & $-0,07803933$ \\
\hline VS & $-0,8446387$ & $-0,47810031$ \\
\hline POB & 0,9597658 & $-0,25292473$ \\
\hline
\end{tabular}

Fuente: elaboración de los autores.

cuando la población aumenta, de igual modo lo hace el tejido urbano continuo 
y disminuyen las coberturas relacionadas con los cuerpos de agua (canales y lagos-lagunas) y vegetación alóctona. Esta primera componente reúne las variables que muestran los procesos de transformación de las coberturas naturales en artificiales por procesos de urbanización.

En la segunda componente, las variables con más peso y de valores positivos son vegetación acuática (VA) y tejido urbano discontinuo (TUD) (figura 7 y tabla 8). La cobertura zonas verdes urbanas (ZVU) presenta una fuerte relación negativa con el componente $2(<-0,75)$, mostrando nuevamente una correlación inversa entre una cobertura artificial de origen antrópico ubicada alrededor del tejido urbano y en la cual predomina el pasto, siendo esta una especie invasora que ha colonizado los cuerpos de agua, transformando la vegetación que se encuentra presente. Esta segunda componente representa cambios de coberturas por invasión de especies foráneas (acción antrópica indirecta).

\section{Discusión}

De acuerdo con los resultados obtenidos, se observa que las coberturas lagos y lagunas (LL), vegetación acuática (VA), pastos limpios (PL), vegetación secundaria (VS) y canales (C) han sufrido conversiones, principalmente hacia coberturas pertenecientes a territorios artificializados (ZVU y TUC), siendo específicamente el tejido urbano continuo el de mayor crecimiento entre los años 1993 a 2015 (32,7 ha), mientras que el cuerpo de agua (LL) perdió la totalidad de su área, transformándose en vegetación acuática. Lo anterior concuerda con el estudio realizado por Ordóñez et al. (2016), quienes analizaron los cambios en las coberturas de tres humedales del Distrito Capital (Santa María del Lago, Córdoba y Juan Amarillo), en los cuales las coberturas espejo de agua y zonas pantanosas fueron reemplazadas por $\mathrm{co}^{-}$ berturas vegetales de humedales, y las zonas verdes urbanas fueron convertidas en tejido urbano, alcanzando para el año 2015 un $79 \%$ del área total de la zona de estudio.

En otros humedales de Bogotá, se presentan situaciones similares en cuanto a la reducción del área en la cobertura de agua, como es el caso del humedal Capellanía (localidad de Fontibón), el cual pasó de tener 16,18 ha en el año 1979 a 0,92 ha en 2008 (Cardona \& Pinilla, 2015); en los humedales de la localidad de Kennedy (Techo, Burro y Vaca), el área de estos en 1949 era de 98 ha, las cuales fueron reduciéndose hasta las 24,5 ha en 2014 (Mayorga, 2016). Para el municipio de Soacha, particularmente en el humedal Tierra Blanca, se evidencian procesos de urbanización (inicialmente parcelas para agricultura, las cuales posteriormente se convirtieron en barrios de carácter tanto legal como ilegal) que fueron creciendo hacia el área de influencia del humedal, afectando la estructura y función de este ecosistema (Ruiz Sepúlveda, 2015).

\section{territarias 43}


Según los resultados obtenidos en el análisis de componentes principales, se hace evidente la relación negativa entre las variables TUC y $\mathrm{POB}$ frente a $\mathrm{PL}, \mathrm{C}$, vs y LL, lo que indica que, si el área de urbanización y la población aumenta, las áreas de las coberturas más naturales como vegetación acuática, espejo de agua y canales están disminuyendo. Conclusiones en ese sentido arrojan los estudios de Rojas et al. (2015), quienes al relacionar variables asociadas a procesos de urbanización e índices de naturalidad para seis humedales ubicados en la región de Concepción Metropolitano de Chile, concluyen que los humedales con mayor naturalidad están más lejos de las cabeceras urbanas. De igual manera, Smith y Romero (2009) indican una relación espacial entre las secuencias de cambio en los usos y coberturas del suelo y la reducción de los niveles de calidad ambiental a través del tiempo; los usos de suelo de tipo urbano se asocian con niveles de calidad ambiental media y baja de los humedales.

El aumento del área del TUC alrededor del humedal se produjo principalmente por el desarrollo informal de los barrios Manzanares, La Primavera, Esperanza de Tibanica, El Palmar y Los Olivos I y II, entre la década de los años ochenta y noventa (Secretaría Distrital de Ambiente \& Instituto de Estudios Ambientales, 2006). Esta situación se vio favorecida gracias a la diferencia en los precios del suelo en relación con otros sectores de la ciudad, promoviendo que aquellos que deberían ser destinados para protección fueran ocupados por vivienda informal de baja calidad urbanística, generando una severa transformación en las coberturas y el deterioro del ambiente acuático en el humedal Tibanica (Carvajal, 2015). Este fenómeno es también reportado por Mayorga en 2016 para los humedales de la localidad de Kennedy, ubicada al igual que Bosa al suroccidente de Bogotá, los cuales han perdido cerca 73,5 ha, siendo el desarrollo urbano de origen informal la principal causa de su reducción.

\section{Conclusiones}

Este estudio permitió determinar que los principales procesos de cambio en las coberturas del humedal Tibanica durante el período 1993-2015 corresponden a la conversión de zonas verdes urbanas y tejido urbano discontinuo a la cobertura tejido urbano continuo, siendo esta última la que presentó un mayor crecimiento durante los años analizados. Adicionalmente, se presentó la conversión total de la cobertura lagos, lagunas y ciénagas naturales, a vegetación acuática, siendo esta una de las principales problemáticas del humedal Tibanica, la reducción de su espejo de agua, lo que llevó a la formulación de diferentes instrumentos de gestión, en los cuales se contemplaron acciones de mantenimiento de la franja hídrica, por lo que un porcentaje $(0,12 \%)$ de la vegetación acuática y de zonas verdes 
urbanas $(0,01 \%)$ se convirtió a lagos, lagunas y ciénagas naturales.

Los procesos de transformación desarrollados en el humedal Tibanica guardan relación con el incremento de la urbanización, como se evidencia durante el período 2004-2009, en el cual se genera el mayor crecimiento de la cobertura tejido urbano continuo, debido a la construcción de la urbanización Alamedas del Parque, la cual constituye la única de carácter legal en el sector aledaño al humedal Tibanica.

Frente a la relación entre la actividad antrópica y las dinámicas de las coberturas del humedal, se evidenció a través del ACP que las variables población y tejido urbano continuo presentaron una asociación negativa con aquellas de características más naturales (pastos limpios, canales, vegetación secundaria y lagos, lagunas), lo que implica una reducción de estas últimas debido al crecimiento de la población en la localidad de Bosa, así como de las construcciones, principalmente para vivienda. De igual manera, se observó una asociación negativa entre la vegetación acuática y las zonas verdes urbanas, lo que sugiere que el aumento en esta última favorece los procesos de invasión de especies foráneas, ya que el pasto es el principal constituyente de esta cobertura.

Es importante resaltar que, aunque el humedal Tibanica ha estado expuesto a fuertes procesos de transformación que han llevado a que se encuentre aislado y rodeado por edificaciones, constituye un elemento esencial para el bienestar de los habitantes de la localidad de Bosa, ya que al conservar algunos remanentes de fauna y flora, suministra servicios ambientales asociados a la mejora en la calidad del aire, es un espacio para la contemplación y el desarrollo de actividades vinculadas con la educación ambiental, por lo que este tipo de estudios otorgan elementos para el territorio.

\section{Referencias}

Alcaldía Mayor de Bogotá, \& Departamento Técnico Administrativo del Medio Ambiente (DAMA). (2006). Politica de humedales del Distrito Capital.

Bartlett, M. S. (1937). Properties of sufficiency and statistical tests. Proceedings of the Royal Society of London. Series A - Mathematical and Physical Sciences, 160(901), 268-282. https://doi. org/10.1098/rspa.1937.0109

Bolund, P., \& Hunhammar, S. (1999). Ecosystem services in urban areas. Ecological Economics, 29, 293-301. http:// www.fao.org/uploads/media/Ecosystem_services_in_urban_areas.pdf

Camilleri, S., De Giglio, M., Stecchi, F., \& Pérez-Hurtado, A. (2017). Land use and land cover change analysis in predominantly man-made coastal wetlands: towards a methodological framework. Wetlands Ecology and Management, 25(1), 23-43. https://doi. org/10.1007/s11273-016-9500-4

Cardona, C. M., \& Pinilla, M. (2015). Análisis cartográfico multitemporal 
de cobertura de agua para humedales interiores en Bogotá D. C. en el periodo de 1996-2008. Estudio de caso: humedales de Córdoba, Capellanía y Santa María del Lago. Revista Ingenieros Militares, (10), 23.

Carvajal, N. (2015). Producción del espacio bogotano, la segregación socio-espacial en la zona periurbana. Bollettino della Società Geografica Italiana, VIII(XIII), 9-20. http://societageografica.net/ wp/wp-content/uploads/2016/08/ carvajal_spagnolo.pdf

Chuvieco, E. (1995). Fundamentos de teledetección espacial. https://doi. org/23.304-1995

Chuvieco, E. (2016). Fundamentals of satellite remote sensing. An environmental approach. Taylor \& Francis Group. https://doi.org/10.1201/b19478v

Contraloría de Bogotá (СB). (2010). Informe de auditoría gubernamental con enfoque integral modalidad especial a la gestión ambiental (planes de manejo ambiental, de mejoramiento, de recuperación y administración) de los bumedales Tibanica, Techo, Burro, La Vaca, Meandro del Say y Capellania.

Costanza, R., D’Arge, R., De Groot, R., Farber, S., Grasso, M., Hannon, B., ... Van den Belt, M. (1997). The value of the world's ecosystem services and natural capital. Nature, 287(1), 253-260. https://doi.org/10.1016/ s0921-8009(98)00020-2

Davidson, N. C. (2014). How much wetland has the world lost? Long-term and recent trends in global wetland area. Marine and Freshwater Research, 65(10), 934. https://doi. org/10.1071/MF14173

Departamento Administrativo de la Defensoría del Espacio Público (DADEP). (2017). Reporte técnico de indicadores de espacio público. http://observatorio.dadep.gov.co/sites/default/files/ Reporte-tecnico-2-2017.pdf

Franco, R. (2016). Análisis multitemporal vectorial en ArcGIS. Miniguia de caso. https://mixdyr.files.wordpress. com/2016/07/analisis_multitemporal_vectorial_arcgisdesktop.pdf

Hoyos, L., Cabido, M., \& Cingolani, A. (2018). A multivariate approach to study drivers of land-cover changes through remote sensing in the Dry Chaco of Argentina. ISPRS International Journal of Geo-Information, 7(170), 1-15. https://doi.org/10.3390/ijgi7050170

Ideam (2010). Leyenda nacional de coberturas de la tierra. Metodologia Corine Land Cover adaptada para Colombia. Escala 1: 100000 . Instituto de Hidrología, Metereología y Estudios Ambientales.

Jiang, W., Wang, W., Chen, Y., Liu, J., Tang, H., Hou, P., \& Yang, Y. (2012). Quantifying driving forces of urban wetlands change in Beijing City. Journal of Geographical Sciences, 22(2), 301-314. https://doi.org/10.1007/ s11442-012-0928-z territarias 43 21 
Lobo Guerrero, A. (1992). Geología e hidrogeología de Santafé de Bogotá y su sabana. En Sociedad Colombiana de Ingenieros \& Sociedad Colombiana de Geotécnia (Eds.), VII Jornadas Geotécnicas de la Ingeniería de Colombia (pp. 1-20). http://www.logemin. com/eng/Download/pdf/16_Geologia_hidrogeologia_Sabana_Bogota. pdf

Mayorga, J. M. (2016). El proceso de urbanización de los humedales de Kennedy en Bogotá, Colombia: una perspectiva normativa. Papeles de Coyuntura, 42, 14-53. http://www.papelesdecoyuntura.com/PDF/Papeles de CoyunturaN-42.pdf

Millenium Ecosystem Assessment. (2005). Ecosystems and buman well-being: wetlands and water synthesis. World Resources Institute. https://doi.org/10.1017/ CBO9781107415324.004

Ministerio de Ambiente, Vivienda y Desarrollo Territorial. (2008). Politica de Gestión Ambiental Urbana. http:// www.minambiente.gov.co/images/ AsuntosambientalesySectorialyUrbana/pdf/Politìcas_de_la_Dirección/ Politica_de_Gestion_Ambiental_Urbana.pdf

Ministerio del Medio Ambiente (MMA). (2002). Política Nacional para Humedales Interiores de Colombia. http:// www.minambiente.gov.co/images/

\section{tersitarias 43}

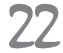
sistemicos/pdf/Normativa/Politicas/ polit_nal_humedales_int_colombia. pdf

Montoya, D. M., \& Reyes, G. A. (2005). Geología de la Sabana de Bogotá. Ministerio de Minas y Energía-Instituto Colombiano de Geología y Minería (Ingeominas). https://choconta.files. wordpress.com/2007/12/informe_ geologia_sabana_bta.pdf

Moreno, J. M. (1995). Estudio geológico y geoomorfológico del sector del "humedal Tibanica"-Potrero Grande, limite entre los municipios de Soacha y el Distrito Capital, Sabana de Bogotá.

Mosquera, S. L., Nieto, O., \& Tapia, C. (2015). Humedales para la gente: visiones desde lo local. Instituto de Investigación de Recursos Biológicos Alexander von Humboldt.

Olsen, S. B. (2000). Educating for the governance of coastal ecosystems: the dimensions of the challenge. Ocean and Coastal Management, 43(4-5), 331-341. https://doi.org/10.1016/ S0964-569l(00)00031-4

Ordóñez, L., González, R., \& Cardona, C. (2016). Conectividad entre los humedales Santa María del Lago, Córdoba, Juan Amarillo y los demás elementos de la estructura ecológica principal en la cuenca urbana del río Salitre, Bogotá D. C. Revista de topografía: Azimut, 7(1), 52-59. https://revistas. udistrital.edu.co/index.php/azimut/ article/view/11450

Pardo, C. E., \& Del Campo, P. C. (2007). Combinación de métodos factoriales 
y de análisis de comglomerados. Revista Colombiana de Estadistica, 30(2), 231-245. http://www. scielo.org.co/scielo.php? pid =S0120$17512007000200006 \&$ script $=s c i$ arttext\&tlng $=$ en

Pedersen, E., Weisner, S. E. B., \& Johansson, M. (2019). Wetland areas' direct contributions to residents' well-being entitle them to high cultural ecosystem values. Science of the Total Environment, 646, 1315-1326. https://doi. org/10.1016/j.scitotenv.2018.07.236

R Core Team. (2017). R: a language and environment for statistical computing. $R$ Foundation for Statistical Computing (Vol. 1). https://doi. org/10.1007/978-3-540-74686-7

Ramsar (2018a). Humedales: esenciales para un futuro urbano sostenible. www. ramsar.org/about/the-scientific-technical-review-panel

Ramsar (2018b). Los humedales urbanos: terrenos valiosos, no terrenos baldios. www.worldwetlandsday.org/es/

Rebelo, L. M., Finlayson, C. M., \& Nagabhatla, N. (2009). Remote sensing and GIS for wetland inventory, mapping and change analysis. Journal of Environmental Management, 90(7), 2144-2153. https://doi. org/10.1016/j.jenvman.2007.06.027

Rojas, C., Pino, J., Basnou, C., \& Vivanco, M. (2013). Assessing land-use and -cover changes in relation to geographic factors and urban planning in the metropolitan area of Concepción
(Chile). Implications for biodiversity conservation. Applied Geography, 39, 93-103. https://doi.org/10.1016/j. apgeog.2012.12.007

Rojas, C., Sepúlveda Zúñiga, E., Barbosa, O., Rojas, O., \& Martínez, C. (2015). Patrones de urbanización en la biodiversidad de humedales urbanos en Concepción Metropolitano. Revista de Geografia Norte Grande, 2015(61), 181-204. https://doi.org/10.4067/ S0718-34022015000200010

Ruiz Sepúlveda, D. C. (2015). Análisis histórico y prospectiva del humedal Tierra Blanca. Perspectiva Geográfica, 19(1), 125. https://doi. org/10.19053/01233769.3326

Secretaría Distrital de Ambiente (SDA), \& Instituto de Estudios Ambientales (IDEA). (2006). Plan de Manejo Ambiental del Parque Ecológico Distrital Humedal Tibanica. http://ambientebogota.gov.co/documents/10157/ a 839 bbl l- 389 b- 4225 - bc 03 a74lf54eb529

Smith, P., \& Romero, H. (2009). Efectos del crecimiento urbano del área metropolitana de Concepción sobre los humedales de Rocuant-Andalién, Los Batros y Lenga. Revista de Geografía Norte Grande, (43), 81-93. https://doi.org/10.4067/S071834022009000200005

Van der Hammen, T., Stiles, F. G., Rosselli, L., Chisacá, M. L., Camargo Ponce de León, G., Guillot Monroy, G., Useche Salvador, Y., Rivera Ospina, territarias 43 


$\left|\begin{array}{c}\text { D. (2008). Protocolo de recuperación } \\ \text { y rehabilitación ecológica de humedales } \\ \text { en centros urbanos. Secretaría Distrital } \\ \text { de Ambiente. } \\ \text { Zhang, W., Jiang, J., \& Zhu, Y. (2015). } \\ \text { Change in urban wetlands and their } \\ \text { cold island effects in response to rapid } \\ \text { urbanization. Chinese Geographical } \\ \text { Science, 25(4), 462-471. https://doi. } \\ \text { org/10.1007/s11769-015-0764-Z }\end{array}\right|$

territarias 43

24 